\title{
Numerical Optical Low-Coherence Holographic Microscopy
}

\author{
Cuche E., *Poscio P., Depeursinge Ch.
}

Institut d'Optique Appliquée, EPFL, CH-1015 Lausanne, Switzerland.

*Institut de Recherche en Ophtalmologie (IRO), CH-1950 Sion 4, Switzerland.

\begin{abstract}
:
The Optical Low-Coherence Holographic Microscopy (OLCHM) is a three dimensional imaging method which mixes the concepts of low coherence holography and holographic microscopy. We present here a numerical application of this method that consists of recording a digital hologram on a CCD camera, the reconstruction of which is performed numerically by means of a Fast Fresnel Transform algorithm. The transverse resolution of the reconstructed images mainly depends on the resolution limit of the optics components used $(\approx 1 \mu \mathrm{m})$. As a consequence of the low coherence, the depth resolution is reduced to about $25 \mu \mathrm{m}$.
\end{abstract}

\section{INTRODUCTION:}

In conventional optical holography, the light intensity in the hologram plane $I_{H}(x, y)$ is recorded on a photographic plate. After development, the hologram is illuminated with a wave identical to the reference and the transmitted wave front contains four terms:

$$
\Psi(x, y)=R R^{2}+R O^{2}+R^{2} O^{*}+R^{2} O
$$

Where $O$ and $R$ are the complex amplitudes of the object and reference waves respectively and $O, R$ their modulus. The two first terms correspond to the zero order diffraction, the third term to the real image and the fourth to the twin image [1]. If the hologram has been recorded according to the Fresnel condition:

$$
d^{3}>\frac{\pi}{4 \lambda}\left[(\xi-x)^{2}+(\eta-y)^{2}\right]^{2}{ }_{\text {max }}
$$

with $\lambda$ the wavelength and $d$ the distance between the hologram plane $0 x y$ and the object plane $0 \xi \eta$, the transmitted wave front $\Psi(\xi, \eta)$ can be calculated at a distance $d$ from the hologram with the Fresnel-Kirchoff integral:

$$
\begin{aligned}
\Gamma(\xi, \eta)= & A \exp \left(\frac{i \pi}{\lambda d}\left(\xi^{2}+\eta^{2}\right)\right) \\
& F\left[I_{H}(x, y) \exp \left(\frac{i \pi}{\lambda d}\left(x^{2}+y^{2}\right)\right)\right]_{\frac{\xi}{\lambda d} \frac{\eta}{\lambda d}}
\end{aligned}
$$

Where $F$ is the Fourier transform operator. In our case, the hologram intensity is recorded on a CCD camera and the reconstruction must be performed by means of a discrete formulation [2] of equation (3):

$$
\begin{gathered}
\Gamma(m \Delta \xi, n \Delta \eta)=\exp \left(\frac{i \pi}{\lambda d}\left(m^{2} \Delta \xi^{2}+n^{2} \Delta \eta^{2}\right)\right) \\
D F T\left[I_{H}(k, l) \exp \left(\frac{i \pi}{\lambda d}\left(k^{2} \Delta x^{2}+l^{2} \Delta y^{2}\right)\right)\right]_{m, n}
\end{gathered}
$$

Where $\mathrm{k}, \mathrm{l}, \mathrm{m}, \mathrm{n}=1 . . \mathrm{N}$ are integers and $\Delta \xi, \Delta \eta$ are the sampling intervals after the discrete Fresnel Transform. DFT is the Discrete Fourier Transform operator which can be very quickly computed using FFT algorithms. Finally
$I_{H}(k, l)$ is a matrix of $N^{2}$ elements which results from the sampling of $I_{H}(x, y)$ :

$$
\begin{aligned}
I_{H}(k, l)=I_{H}(x, y) \operatorname{rect}\left(x / L_{x}, y / L_{y}\right) \\
\qquad \sum_{k=1 l=1}^{N x} \sum_{l=1}^{N y} \delta(x-k \Delta x, y-l \Delta y)
\end{aligned}
$$

Where $L_{x}$ and $L_{y}$ are the dimensions of the CCD array and $\Delta x, \Delta y$ its pixel sizes. According to equations (3) and (4), the pixel sizes in the image and hologram plane are connected as follow [3]:

$$
\Delta \xi=\frac{\lambda d}{N \Delta x}=\frac{\lambda d}{L x} \quad \Delta \eta=\frac{\lambda d}{N \Delta y}=\frac{\lambda d}{L y}
$$

Thus, for a given CCD camera, if the object size is greater than the quantity $L_{\xi}=N \Delta \xi$, aliasing problems will appear and if a detail of the object is smaller than $\Delta \xi$ it will not be reproduced by the numerical reconstruction.

\section{METHODS:}

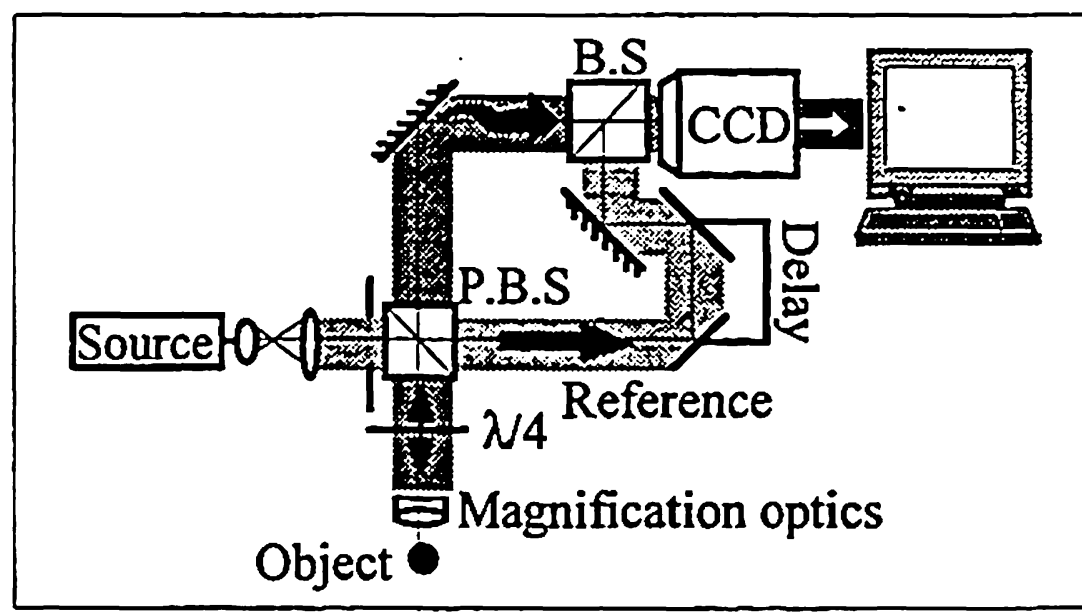

Fig. I Schematic of the numerical OLCHM imaging setup (PBS: Polarizing Beam Splitter)

The experimental setup designed is shown in figure 1. After enlargement of the spot size, a polarizing beam splitter (PBS) separates the incident light. On one hand, the horizontal component of the polarized beam crosses the PBS and forms the reference wave whose incidence angle $\theta$ on the CCD can be controlled by changing the orientation of a mirror. On the other hand, the vertical component is reflected by the PBS in the direction of the object and a quarter wave plate ensures that the back scattered light crosses the PBS and reaches the CCD after reflection on a mirror. A frame grabber captures the hologram from a standard CCD camera with $512 \times 768$ pixels of $6.8 \times 6.8 \mu \mathrm{m}^{2}$ area. Finally the holographic reconstruction is performed by a computer. For microscopic investigations magnification optics can be installed between the object and the PBS. Two kinds of configurations have been studied here; a single lens of $30 \mathrm{~mm}$ focal length and $5 \mathrm{~mm}$ diameter and an microscope objective of magnifica- 
tion $\times 20$ and 0.35 numerical aperture. Two kinds of sources have been used, an He-Ne laser for preliminary results and a Ti:sapphire laser with $\sim 80$ fs pulse duration for low-coherence investigations

\section{RESULTS AND DISCUSSION:}

Figure 2 shows a typical result of numerical OLCHM. The hologram (fig. 2a) has been recorded with an He-Ne laser and the groups 2 and 3 of the USAF test target as object. No magnification optics were used here. Figure $2 b$ shows the corresponding numerically reconstructed image using algorithm (4) with a reconstruction distance $d=135$ $\mathrm{mm}$. It is important here to note that $d$ must be equal to the optical path and doesn't match the effective distance separating the object and the $C C D$, essentially because of the presence of beam splitters. The four different terms of the

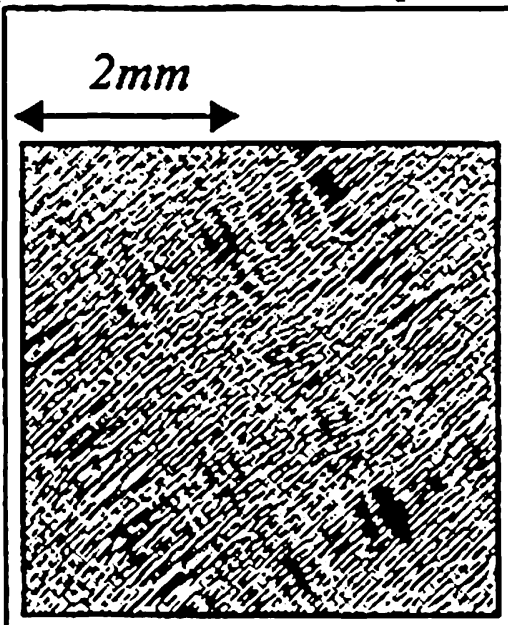

a)

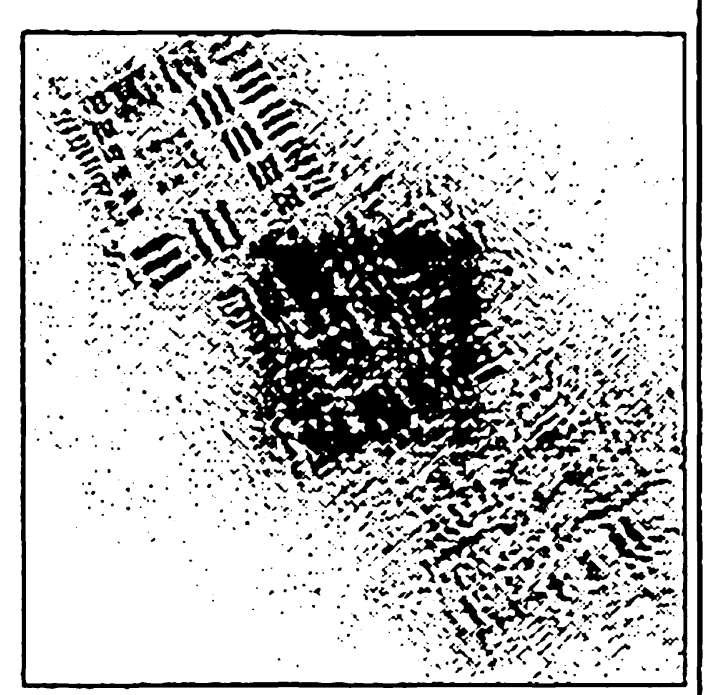

b)
Fig. 2 Typical result of numerical OLCHM.

a) The hologram b) The reconstructed image.

transmitted wave $\Psi$ mentioned in the introduction (equ. 1) are clearly observable in figure $2 b$. In the centre of the image appears the zero order diffraction intensity inside which a term proportional to the square of the object wave modulus is distinguishable. In the top left comer one can observe the real image and symmetrically with respect to the centre, a blurred twin image. These three terms are spatially separated because an angle $\theta$ has been introduced between the reference and object waves at their incidence on the CCD. With these experimental conditions, the smallest observable object is the element number 6 of group 3 of the USAF target. This means a resolution of around $35 \mu \mathrm{m}$ which is comparable to the 21 $\mu \mathrm{m}$ calculated from equation (6) for $d=135 \mathrm{~mm}, \lambda=632.8$ $\mathrm{nm}$ and the parameters of the CCD as specified above.

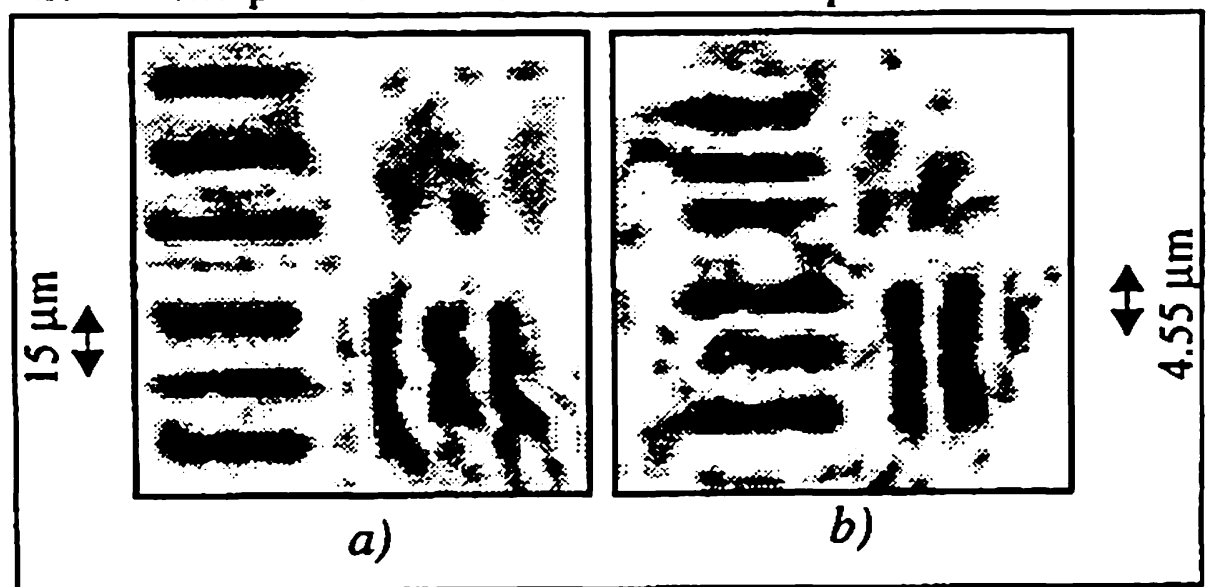

Fig. 3 Resolution limit: a) obtained with a single lens b) obtained with a microscope objective

Images of figures $3 \mathrm{a}$ and $3 \mathrm{~b}$ have been obtained with $\mathrm{a}$ single lens and a microscope objective as magnification optics respectively. In the first case, we can see the second element of the group 6 and in the second case the sixth element of the group 7. This means a resolution of about $7.5 \mu \mathrm{m}$ for the single lens and $2.25 \mu \mathrm{m}$ for the objective. These two results are quite equal to the theoretical of 5 $\mu \mathrm{m}$ and $2.2 \mu \mathrm{m}$ resolutions limits obtained by application of the Rayleigh criterion for these two optics.

Images in figure 4 have been reconstructed from holograms recorded with the Ti:sapphire laser and the character " 4 " of the title of the fourth group of the USAF test target as object. Firstly, the delay device was set on the ideal position for which the optical paths are the same along the reference and object arms. Then six holograms have been recorded after displacement with $10 \mu \mathrm{m}$ steps of the delay device backward and forward its initial position. We can clearly see that the image disappear along the

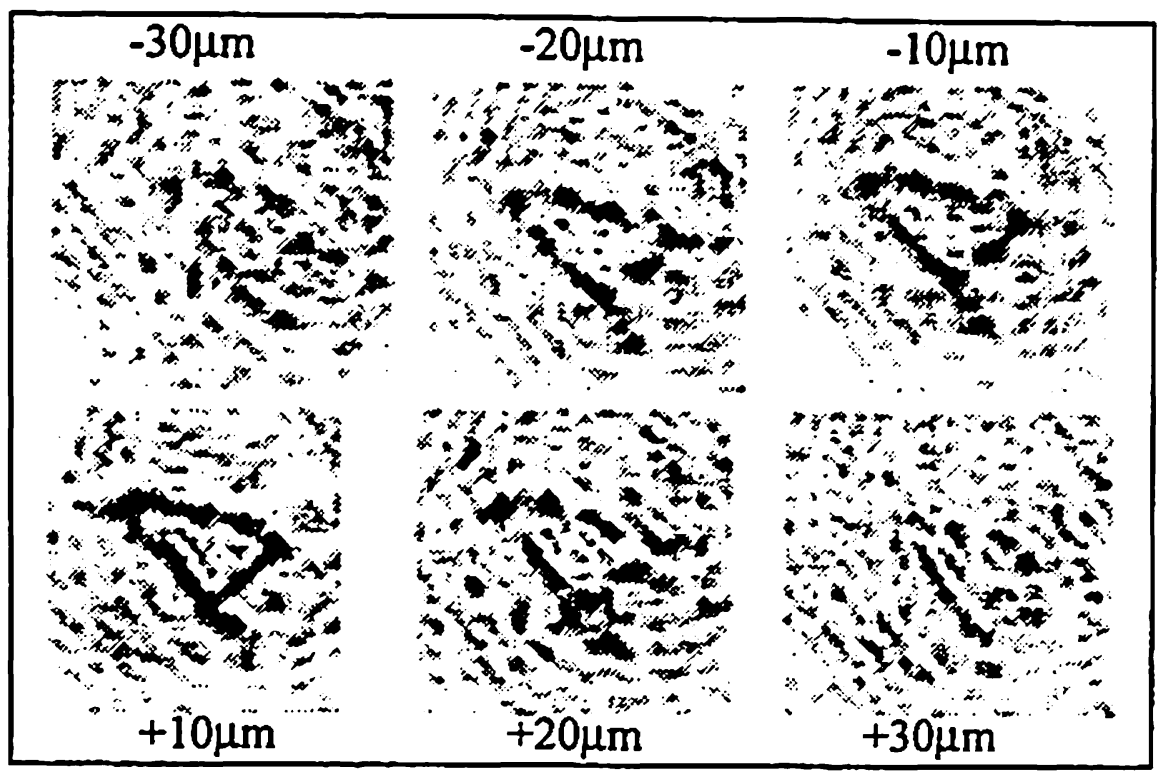

Fig. 4 Depth resolution: Reconstructed images for six different positions of the delay device.

both direction for displacement higher than $20 \mu \mathrm{m}$. Thus the depth resolution can be estimated to $25 \mu \mathrm{m}$ which corresponds to the length of our $80 \mathrm{fs}$ pulse duration.

\section{CONCLUSION:}

The numerical reconstruction algorithm and the experimental setup have been successfully tested with an USAF test target. The transverse resolution is limited by the magnification optics used via the Rayleigh criterion and the depth resolution is equal to coherence length of the source. With a specialized hardware the numerical reconstruction could be performed at the video frequency. This opens a wide range of exciting perspectives for new applications of low-coherence holography.

\section{ACKNOWLEDGMENTS:}

This work was supported by the Swiss National Foundation (grant FN 2100-042323.94/1) and the UNIL-EPFL grant (New optical methods in ophtalmology). The authors thank Frédéric Bevilacqua and Olivier Coquoz for interesting remarks about the manuscript.

\section{REFERENCES}

[1]J. W. Goodman, An Introduction to Fourier Optics and Holography, (McGraw-Hill, New York, 1968).

[2]U. Schnars, W. Jüptner, Applied Optics, 33 (1994), 179.

[3]L. P. Yaroslavskii, N.S. Merzlyakov, Methods of Digital Holography (Consultant Bureau, New York, 1980). 